\title{
Pierre Bourdieu: Una mirada etnográfica Aportes al diseño sobre la cultura material y la educación
}

Proyecto de Investigación 15.1 Equipo de Investigación

Patricia Vargas y Laura Colabella por Facultad de Diseño y Comunicación, Universidad de Palermo (ARG) y el Centro de Antropología Social del Instituto de Desarrollo Económico y Social, CAS-IDES (ARG)

Línea de Investigación 15. Etnografía, cultura material y educación: Reflexiones y aportes al diseño

\section{Resultados publicados en el [Cuaderno 88]}

Cuaderno del Centro de Estudios de Diseño y Comunicación No88. (2021) Aportes al análisis de las prácticas culturales contemporáneas de la Argentina reciente, desde la perspectiva de Pierre Bourdieu. Coordinación Laura Colabella y Patricia Vargas (UP-CAS_IDES). Programa de Investigación de la Facultad de Diseño y Comunicación. Centro de Estudios en Diseño y Comunicación. Facultad de Diseño y Comunicación. Universidad de Palermo. Año XXI, Febrero 2021, Buenos Aires, Argentina. ISSN: 1668-0227.

Los resultados de este Proyecto de Investigación fueron evaluados por Ileana Grisel Adissi y su Dictamen se transcribe a continuación:

\section{Síntesis de la Evaluación}

Actualidad de la temática (Vigencia, Aplicación) / Notable Nivel Alcanzado (Profundidad, Consistencia, Jerarquía) / Buena

Diversidad del enfoques (Pluralidad, Participación) / Notable

Calidad (Problemática, Análisis, Tratamiento) / Buena

Contribución al avance del Diseño (Fortaleza) / Buena

\section{Fundamentos y Comentarios}

El Cuaderno 88 reúne una serie de trabajos sólidos y relevantes. Resulta de especial interés la articulación entre teoría y empiria, puesto que el recorrido propuesto permite adentrarse por diversos campos de la experiencia, objetos, relaciones sociales, prestando atención esmerada a los aspectos descriptivos de cada uno de los entornos, al tiempo que presenta 
conceptualizaciones y discusiones en un nivel elevado de abstracción. El que varios artículos estén escritos en primera persona, dando lugar a la voz singular de quien investiga en su experiencia como investigador/a refuerza el interés de la propuesta.

Ahora bien, el Cuaderno en su conjunto presenta cierta falta de focalización que vuelve difícil de precisar el sentido de la propuesta en términos globales. En principio el propio título ("Aportes...") no explicita la relación con el Diseño como área de conocimiento, además de que la redundancia ("contemporáneas"/"reciente") diluye la explicitación del recorte, de por sí muy amplio. Este carácter difuso del título tiende a repetirse en el prólogo, que si bien se propone unificar la presentación, podría ganar más claridad al argumentar la pertinencia de la inclusión de los distintos artículos para el tratamiento de un mismo y único problema. Ta vez se podría haber optado por un formato convencional en términos de pregunta- problema y objetivos para asentar el interés de conocer los distintos artículos, en la medida en que todos ellos aportan a la consolidación de una misma área, justificando simultáneamente el aporte al campo del diseño.

Sumado a lo anterior, en algunos artículos la perspectiva de Pierre Bourdieu pareciera haber sido introducida a posteriori de un desarrollo previo, en lugar de haber orientado la indagación empírica. Este carácter aparentemente forzado de la conceptualización admite ser subsanado mediante la reescritura, y nuevamente apelando al establecimiento inicial de una pregunta que requiera, para poder ser respondida, tanto de los aportes empíricos como de los teóricos. Porque no queda claro si se apunta a homenajear a Pierre Bourdieu (tal como se explicita inicialmente $-\mathrm{y}$ en este caso, en qué consistiría dicho homenaje, puesto que este término es polisémico, y por qué resultaría conveniente o apropiado realizarlo), a visualizar ciertas dimensiones empíricas de la mano de algunos textos particulares del autor, a establecer un área de interés en la interfase entre distintos campos de estudio, u alguna otra intencionalidad no declarada. Por otra parte, para potenciales lectores que no conozcan al autor tal vez sería enriquecedor incluir una reseña sobre el mismo, que permita remitir las distintas obras mencionadas en las referencias teóricas de cada uno de los artículos a una genealogía mayor, aclarando su sentido.

En síntesis, la obra se muestra promisoria pero pareciera requerir un estadio superior de reescritura para reforzar su direccionalidad y consistencia interna. 